\title{
Importance of multi-mesonic fusion processes on (strange) antibaryon production
}

\author{
C. GREINER \\ Institut für Theoretische Physik, Universität Giessen, Heinrich-Buff-Ring 16, D-35392 Giessen, \\ Germany
}

\begin{abstract}
Sufficiently fast chemical equilibration of (strange) antibaryons in an environment of nucleons, pions and kaons during the course of a relativistic heavy ion collision can be understood by a 'clustering' of mesons to buildt up baryon-antibaryon pairs. This multi-mesonic (fusion-type) process has to exist in medium due to the principle of detailed balance. Novel numerical calculations for a dynamical setup are presented. They show that - at maximum SPS energies - yields of each antihyperon specie are obtained which are consistent with chemical saturated populations of $T \approx 150-160 \mathrm{MeV}$, in line with popular chemical freeze-out parameters extracted from thermal model analyses.
\end{abstract}




\section{BRIEF OVERVIEW ON ANTIHYPERON PRODUCTION}

Strangeness enhancement has been predicted a long time ago as a potential probe to find clear evidence for the temporary existence of a quark gluon plasma (QGP) in relativistic heavy ion collisions. A strong experimental effort has been made since and is still made for measuring strange particle abundancies in experiments at Brookhaven and at CERN (for a short recent review see [1]). In particular because of high production thresholds in binary hadronic reaction channels antihyperons had been advocated as the appropriate QGP candidates [2]. Indeed, a satisfactory picture of nearly chemically saturated populations of antihyperons has been experimentally demonstrated over the last years with the $\mathrm{Pb}+\mathrm{Pb}$ experiments NA49 and WA97 at CERN-SPS. For this statement, of course, a quantitative, theoretical analysis by employing a thermal (or 'statistical') model has to be invoked by fitting the thermodynamical parameters to the set of individual (strange) hadronic abundancies [3].

On the other hand, already since the first measurements with the lighter ions have been undertaken in the early nineties, the theoretical description of the antibaryon production within hadronic transport schemes in comparison to these data faced some severe difficulties. Some phenomenological motivated attempts to explain a more abundant production of antihyperons within a hadronic transport description [4] had been proposed like the appearance of color ropes, the fusion of strings, the percolation of strings, or the formation of high-dense hadronic clusters. The underlying mechanisms, however, have to be considered exotic and to some extent ad hoc, their purpose lies mainly to create (much) more antibaryon in the very early intial stage of the reaction (compared to simple rescaled $\mathrm{p}+\mathrm{p}$ collisions). To some extent indeed the philosophy behind these mechanisms was to model a precursor of initial QGP formation within a hadronic transport scheme. On the other hand, in most of the transport calculations a dramatic role of subsequent antibaryon annihilation is observed, which, in return, has to be more than counterbalanced by these more exotic, initially occuring mechanisms. Large annihilation rates result if the free-space cross section is employed. The situation seems even more paradox with respect to the fact that the chemical descrip-

tion within thermal models works indeed amazingly well for the antihyperons and being, of course, completely nondependent on the (large) magnitude of the annihilation cross section. For all of this reasons the theoretical and dynamical understanding of the production of (strange) antibaryons has remained a delicate and challenging task [5]. 


\section{FIGURES}
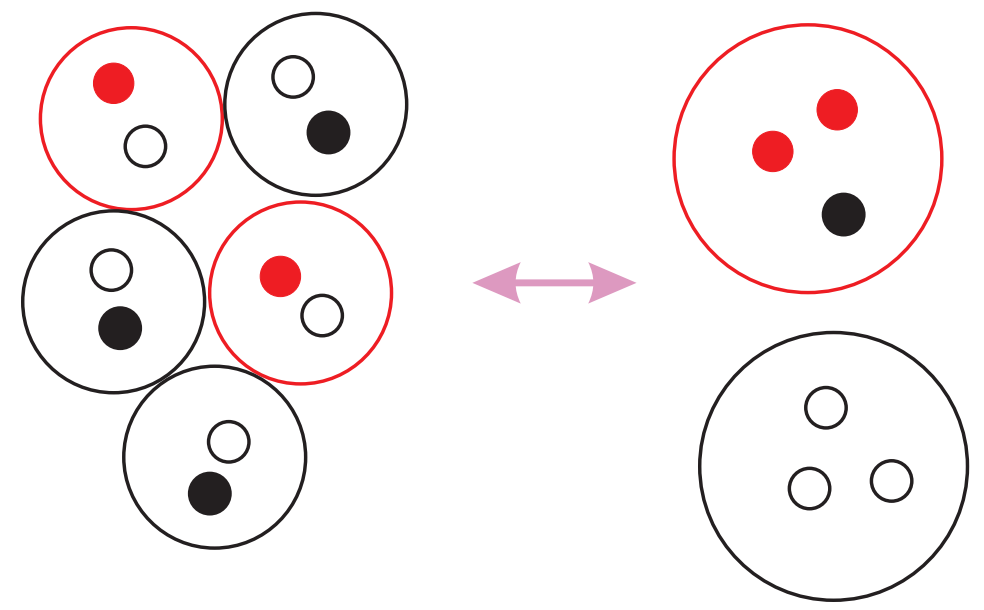

FIG. 1. Schematic picture for the multi-mesonic fusion-like reaction $3 \pi+2 K \leftrightarrow \bar{\Xi}+N$.

As we will demonstrate, a correct incorporation of the baryonic annihilation channels had actually not been done consistently. We have conjectured recently that a sufficiently fast redistributions of strange and light quarks into (strange) baryon-antibaryon pairs should be achieved by multi-mesonic fusion-type reactions of the type

$$
n_{1} \pi+n_{2} K \leftrightarrow \bar{Y}+p
$$

occuring in a moderately dense hadronic system [6] (illustrated in Fig. 1). The beauty of this argument lies in the fact that (at least) these special kind of multi-hadronic reactions have to be present because of the fundamental principle of detailed balance. As the annihilation of antihyperons on baryons is of dramatic relevance, the multi-mesonic (fusion-like) 'backreactions' involving $n_{1}$ pions and $n_{2}$ kaons, where $n_{2}$ counts the number of anti-strange quarks within the antihyperon $\bar{Y}$ ), must, in principle, be taken care of in a dynamical simulation. These reactions are then the most dominant source of production. This crucial fact had been overseen in all of the aforementioned treatments. The underlying reasoning was first raised by Rapp and Shuryak who described the maintenance of nearly perfect chemical equilibrium of antiprotons together with pions and nucleons during the very late stage of the expanding fireball before the particles loose contact [7].

The crucial input, though plausible, is now to assume that the annihilation cross sections for any strange or nonstrange antibaryon on any baryon are approximately the same order as for $N \bar{p}$ at the same relative momenta, i.e. $\sigma_{B \bar{Y} \rightarrow n \pi+n_{Y} K} \approx \sigma_{N \bar{p} \rightarrow n \pi}$, being in the range of 50$100 \mathrm{mb}$ for characteristic and moderately low momenta occuring in an expanding hadronic fireball. The equilibration timescale $\left(\Gamma_{\bar{Y}}\right)^{(-1)} \sim 1 /\left(\sigma_{B \bar{Y}} v_{B \bar{Y}} \rho_{B}\right)$ is to a good approximation proportional to the inverse of the density of baryons and their resonances. Adopting an initial density of 1-2 times normal nuclear matter density $\rho_{0}$ for the initial and thermalized hadronic fireball, the antihyperons do equilibrate on a timescale of $1-3 \mathrm{fm} / \mathrm{c}$ well within the expansion timescale of the late hadronic fireball. Hence, fast chemical equilibration of the antihyperon abundancies is guaranteed by detailed balance with respect to the strong annihilation, the final yields then being independent of the actual size of the (large) annihilation cross section, solving the aforementioned paradox. 
To be quantitative, (some) novel results by solving rate calculations for a dynamical setup are presented in the following. Before turning to their discussion, we will review briefly on some general ideas of the baryon-antibaryon annihilation process, in order to strengthen the one main assumption concerning the general size of its cross section, and on how to come to general master equations.

\section{ANTIBARYON ANNIHILATION AND ITS EFFECTIVE DESCRIPTION BY A MASTER EQUATION}

Antinucleon-nucleon annihilation is the strongest of all strong interaction processes. The strong annihilation of a nucleon and an antinucleon can be thought quantum mechanically as a complete absorbtive scattering process given approximately by the black disk formula $\sigma_{a b s}=\pi(R+\lambda)^{2}$ or by a more sophisticated boundary condition description [8]. For $p+\bar{p}$ annihilation one finds for the 'black disk' radius $R=1.07 \mathrm{fm}$, for which then this decription reproduces very accurately the total inelastic cross section as a function of the beam momentum [8] and also its steep increase and diverging behaviour at low momenta. Hence, the quantum mechanical interpretation of the annihilation (being exotherm) is a picture of complete absorption. One is now tempted to generalize this simple and intuitive picture for all baryon-antibaryon annihilation processes. The only 'free' parameter which then can change is the radius $R$. Again, as it basically reflects the radius of the proton in the case of $p \bar{p}$-annihilation, one would expect that this canonical value of $1 \mathrm{fm}$ is of general validity for all the annihilation processes. Thus it is plausible to assume that the cross section for annihilation between any baryon and antibaryon should be rather the same for the same relative momenta. Indeed, there does exist old data on the total $\bar{\Lambda}+p$ cross section [9] with reasonably large value, although the $\bar{\Lambda}$ momenta was exceeding $4 \mathrm{GeV} / \mathrm{c}$ in all data taken. (These data are not conclusive to really extrapolate dwn to lower momenta, though they give a right indication: The authors of [9] reported an extrapolated formula of $\sigma_{a b s}=47( \pm 10) p_{\Lambda}^{-1 / 2} \mathrm{mb} \mathrm{GeV}^{-1 / 2}$, which is not fully analogue to the black disk formula, but has already a rather correct shape with a sizeable magnitude.)

A more microscopic and quite popular picture, which can decribe quantitatively the complicated final states of individual outgoing mesonic channels, is the concept of two meson doorway states [10] given by

$$
\bar{B}+B \rightarrow M_{1}+M_{2} \rightarrow n_{1} \pi+n_{2} K .
$$

The main assumption is that the annihilation occurs exclusively via two mesons with nearest threshold dominance. $M_{1}$ and $M_{2}$ can be rather highlying resonances and/or hybrid states which come close with their individual threshold. The final decay of these two mesons into the various channels of multiple pions and kaons is then treated statistically and microcanonically. It turns out that such a description describes rather nicely reproduces the available data and can also be generalized straightforwardly for antihyperon annihilation [10]. There do exist a lot of other phenomenological and microscopic descriptions like quark models or flux tube models, which try to describe quantitatively the complicated process of the annihilation in more dynamical terms (for a review see [11]). 
How can one incorporate such complicated processes into a description of transport dynamics? It is clear that as the very microscopy of the annihilation processes is not fully understood, one has to abandon any detailed local modelling, but has to turn to an effective coarse grained description which is guided by physical principles. Following the concepts of relativistic kinetic theory, the microscopic starting point is a Boltzmann-type equation of the form

$$
\begin{aligned}
\partial_{t} f_{\bar{Y}}+\frac{\mathbf{p}}{E_{\bar{Y}}} \nabla f_{\bar{Y}}= & \sum_{\left\{n_{1}\right\} ; B} \frac{1}{2 E_{\bar{Y}}} \int \frac{d^{3} p_{B}}{(2 \pi)^{3} 2 E_{B}} \prod_{\left\{n_{1}, n_{2}\right\}} \int \frac{d^{3} p_{i}}{(2 \pi)^{3} 2 E_{i}}(2 \pi)^{4} \delta^{4}\left(p_{\bar{Y}}+p_{B}-\sum_{\left\{n_{1}, n_{2}\right\}} p_{i}\right) \\
& \left|\left\langle n_{1}, n_{2}|T| \bar{Y} B\right\rangle\right|^{2}\left\{(-) f_{\bar{Y}} f_{B} \prod_{\left\{n_{1}, n_{2}\right\}}\left(1+f_{i}\right)+\prod_{\left\{n_{1}, n_{2}\right\}} f_{i}\left(1-f_{\bar{Y}}\right)\left(1-f_{B}\right)\right\},
\end{aligned}
$$

where

$$
\sigma_{\bar{Y} B}^{\left\{n_{1}\right\}} \equiv \frac{1}{{ }^{6} F l u x^{\prime}} \sum_{\left\{n_{1}\right\}} \prod_{\left\{n_{1}, n_{2}\right\}} \int \frac{d^{3} p_{i}}{(2 \pi)^{3} 2 E_{i}}(2 \pi)^{4} \delta^{4}\left(p_{\bar{Y}}+p_{B}-\sum_{\left\{n_{1}, n_{2}\right\}} p_{i}\right) \mid\left\langle\left.\left\langle n_{1}, n_{2}|T| \bar{Y} B\right\rangle\right|^{2}\right.
$$

corresponds to the total annihilation cross section into the various possible multiple pion states. This form of the collision exactly incorporates the principle of detailed balance. The static fixed point of this equation (together with the usual binary kinetic processes) are thermal and chemical saturated distributions. Without the back reaction channels the equations would only have vanishing distributons as stable fixed points, which is, of course, unacceptable. Hence, the back reactions have to be considered as very basic and important ingredient of the transport description when trying to implement the baryon-antibaryon annihilation processes.

Furthermore it is obvious that the back reactions will guarantee that the (strange) antibaryons become chemical saturated with the pions, kaons and nucleons on a very short timescale. To see this more explicit, and also for the further numerical treatment, one can bring the above Boltzmann equation into a more intuitive form of a master or rate equation. Assuming $v_{\text {rel }} \sigma_{\bar{Y} B}(\sqrt{s})$ to be roughly constant, which is actually a good approximation for the $p \bar{p}$-annihilation, or, invoking a standard description of further effective coarse graining by using thermally averaged cross sections and distributions, and furthermore taking the distributions in the Boltzmann approximantion, the following master equation for the respectively considered antihyperon density is obtained

$$
\frac{d}{d t} \rho_{\bar{Y}}=-\left\langle\left\langle\sigma_{\bar{Y} B} v_{\bar{Y} B}\right\rangle\right\rangle\left\{\rho_{\bar{Y}} \rho_{B}-\sum_{\left\{n_{1}\right\}} \hat{M}_{\left(n_{1}, n_{2}\right)}\left(T, \mu_{B}, \mu_{s}\right)\left(\rho_{\pi}\right)^{n_{1}}\left(\rho_{K}\right)^{n_{2}}\right\},
$$

where the 'back-reactions' of several effectively clustering pions and kaons are incorporated in the 'mass-law' factor

$$
\hat{M}_{\left(n_{1}, n_{2}\right)}\left(T, \mu_{B}, \mu_{s}\right)=\frac{\rho_{\bar{Y}}^{e q .} \rho_{B}^{e q .}}{\left(\rho_{\pi}^{e q}\right)^{n_{1}}\left(\rho_{K}^{e q \cdot}\right)^{n_{2}}} p_{n_{1}} .
$$

Here $p_{n_{1}}$ (which will generally depend on the thermodynamical parameters) states the relative probability of the reaction (1) to decay into a specific number $n_{1}$ of pions and $\rho_{B}$ denotes 
the total number density of baryonic particles. As is well-known, the mass-law factor $\hat{M}$ depends only on the temperature and the baryon and strange quark chemical potentials. $\Gamma_{\bar{Y}} \equiv\left\langle\left\langle\sigma_{\bar{Y} N} v_{\bar{Y} N}\right\rangle\right\rangle \rho_{B}$ gives the effective annihilation rate of the respective antihyperon specie on a baryon.

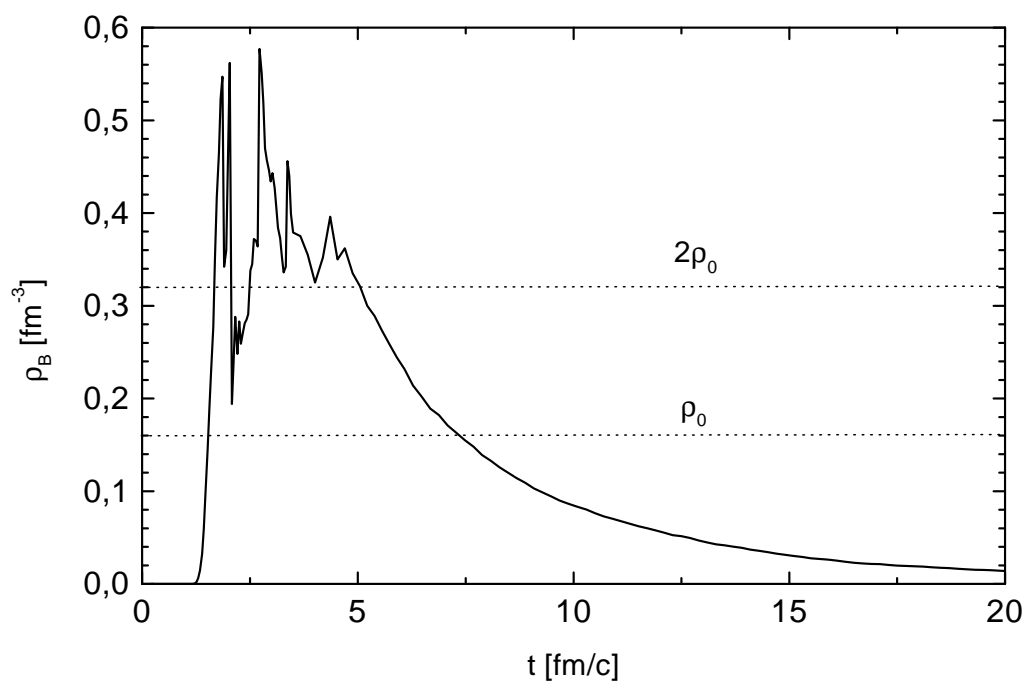

FIG. 2. Time evolution of the (average) net baryon density for midrapidity $|\Delta Y| \leq 1$ and central $\mathrm{Pb}+\mathrm{Pb}$-collision at $160 \mathrm{AGeV}$ obtained within a dynamical transport simulation. Here the amount of baryon number residing still in string-like excitations is explicitely discarded. String-like excitations have disappeared after about $3-4 \mathrm{fm} / \mathrm{c}$, so that from this time on a pure hadronic fireball develops and expands. Its initial net baryon-density starts slightly above $\rho_{B}=2 \rho_{0}$.

For a further manipulation one has to make assumptions for the various abundancies occuring in the master equation. Nonequilibrium inelastic hadronic reactions can explain to a good extent the overall strangeness production seen experimentally: The major amount of the produced kaons at SPS-energies can be understood in terms of still early and energetic non-equilibrium interactions [12]. In Fig. 2 the time evolution of netbaryon density at midrapidiy obtained within a dynamical transport simulation [13] is depicted. All strangeness is being produced still when string-like excitations are governing the dynamics. In the later hadronic stage the number of strange quarks stays more or less constant and can only be redistributed among the various hadrons [14]. Also the pions and nucleons do stay more or less at thermal equilibrium. Refering to the master equation (3), one can then take the pions, baryons and kaons to stay approximately in thermal equilibrium throughout the later hadronic evolution of the collision, the later being modelled to be an isentropic expansion with fixed total entropy content being specified via the entropy per baryon ratio $S / A$ (compare with Fig. 3). (3) has the intuitive form

$$
\frac{d}{d t} \rho_{\bar{Y}}=-\Gamma_{\bar{Y}}\left\{\rho_{\bar{Y}}-\rho_{\bar{Y}}^{e q}\right\} .
$$

The production rate per unit volume $d N_{\bar{Y}} / d t d V$ is given by the non-vanishing, though small value $\Gamma_{\bar{Y}} \rho_{\bar{Y}}^{e q}$. Still this rate is enough to populate the antihyperons to their (very small) 
equilibrium value.

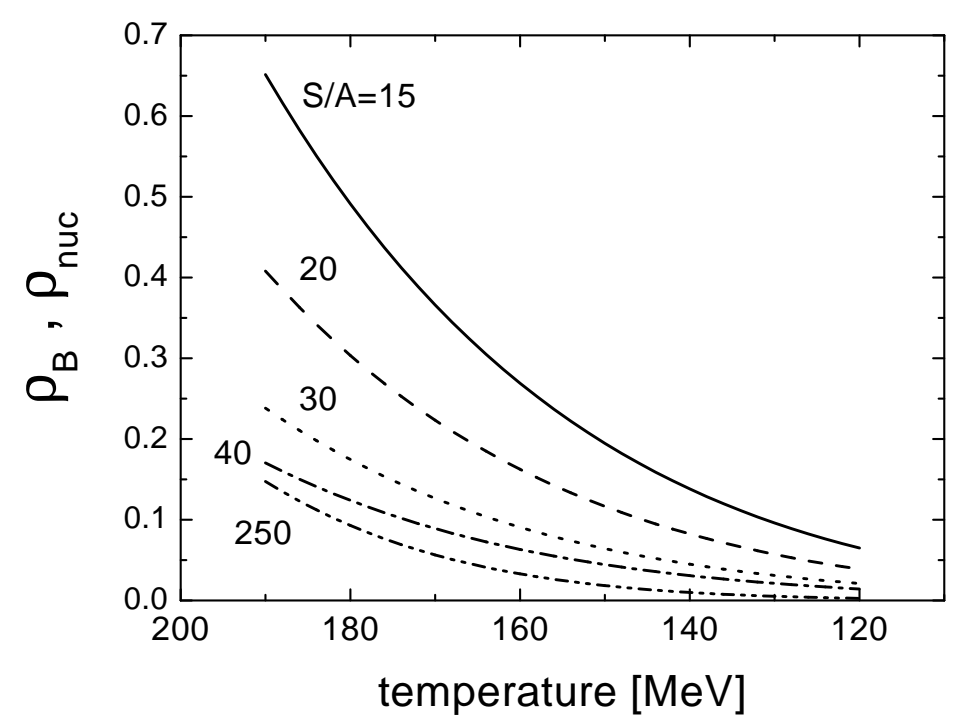

FIG. 3. The netbaryon density $\rho_{B}$ of a chemically fully equilibrated thermal hadronic resonance gas as function of decreasing temperature for the situation of an isentropic expansion, i.e. for various constant entropy/baryon ratios. For the value $S / A=250$ (a situation expected at RHIC) the total baryon number density $\rho_{n u c}$ is plotted instead.

For the calculations solving this master equation (4) one has to employ an 'effective' volume $V(t)$ in order to extrapolate from Fig. 2 to Fig. 3, i.e. simulating the global characteristic of the expansion and the dilution of the baryon density and thus the annihilation rate $\Gamma_{\bar{Y}}(t)$. The 'effective' (global or at midrapidity) volume $V(t)$ is parametrized as function of time by longitudinal Bjorken expansion and including a transversal expansion either with a linear profile

$$
V_{\text {eff,lin }}\left(t \geq t_{0}\right)=\pi(c t)\left(R_{0}+v_{l i n}\left(t-t_{0}\right)\right)^{2}
$$

(taking $v_{l i n}$ as an appropriate parameter to simulate slower or faster expansion) or with an accelerating radial flow

$$
V_{e f f, a c c}\left(t \geq t_{0}\right)=\pi(c t)\left(R_{0}+v_{0}\left(t-t_{0}\right)+0.5 a_{0}\left(t-t_{0}\right)^{2}\right)^{2}
$$

with $R_{0}=6.5 \mathrm{fm}, v_{0}=0.15 c$ and $a_{0}=0.05 c^{2} / \mathrm{fm}$ for the later modeling. 


\section{RESULTS AND IMPLICATIONS}

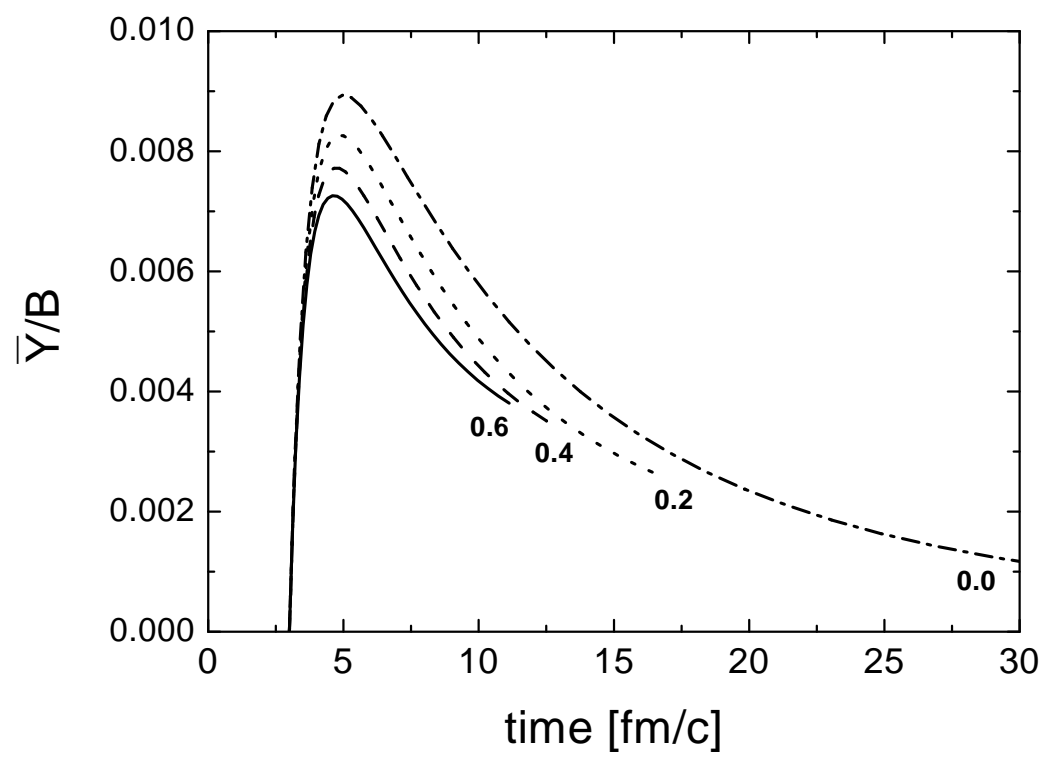

FIG. 4. The anti- $\Lambda$ to baryon number ratio $N_{\bar{\Lambda}} / N_{B}(t)$ as a function of time for various velocity parameters $v_{\text {lin }}$ for the transverse expansion. The entropy per baryon is taken as $S / A=30, t_{0}=3$ $\mathrm{fm} / \mathrm{c}$ and $T_{0}=190 \mathrm{MeV}$.

At starting time $t_{0}$ an initial temperature $T_{0}$ is chosen. ( $T_{0}$ is set to $190 \mathrm{MeV}$ for the SPS and $150 \mathrm{MeV}$ for the AGS situation, while the initial energy densities are then about $1 \mathrm{GeV} / \mathrm{fm}^{3}$.) From (5) or (6) together with the constraint of conserved entropy the temperature and the chemical potentials do follow as function of time and thus also $\rho_{B}(t)$ as well as $\rho_{\bar{Y}}^{e q}(t)$ within the hadronic resonance gas. As a minimal assumption the initial abundancy of antihyperons is set to zero. Equation (4), taking into account the volume dilution, is solved for each specie. For the thermally averaged cross section we take a simple constant value of $\left\langle\sigma_{a n n} v\right\rangle:=\sigma_{0} \equiv 40 \mathrm{mb}$. 


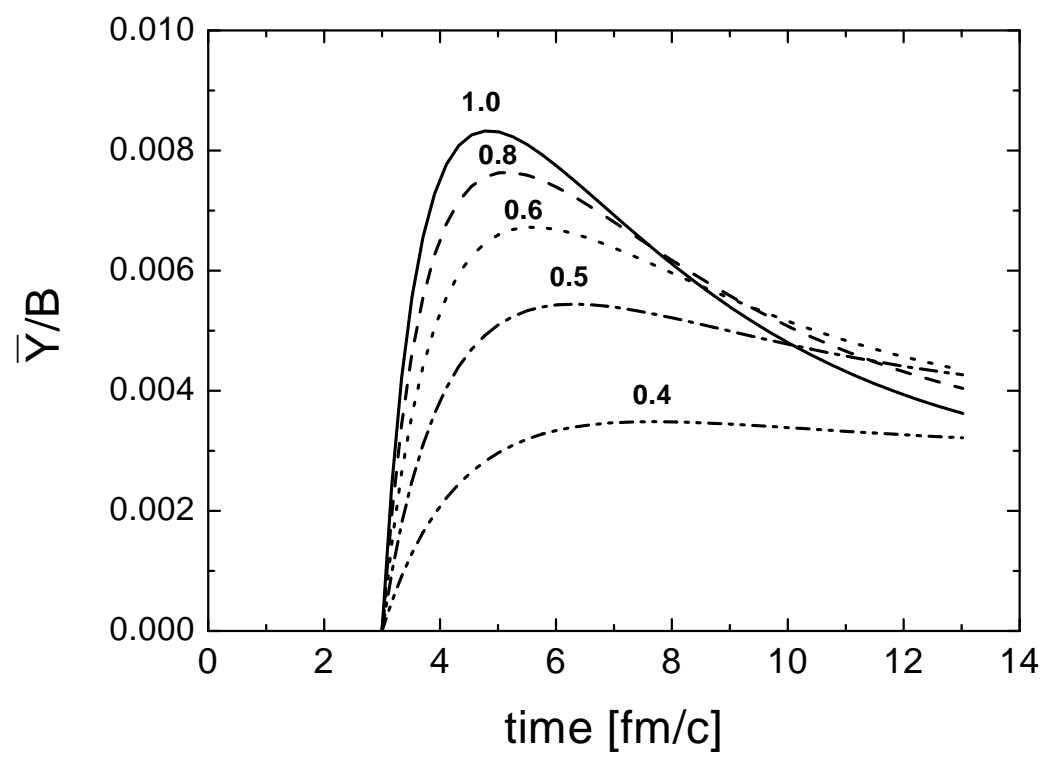

FIG. 5. The anti- $\Lambda$ to baryon number ratio $N_{\bar{\Lambda}} / N_{B}(t)$ as a function of time for various implemented annihiation cross section $\sigma_{\text {eff }} \equiv \lambda \sigma_{0}$. The entropy per baryon is taken as $S / A=30$, $t_{0}=3 \mathrm{fm} / \mathrm{c}$ and $T_{0}=190 \mathrm{MeV}$.

In Fig. 4 the number of $\bar{\Lambda}_{\mathrm{s}}$ (normalized to the conserved net baryon number) as a function of time is depicted. The entropy per baryon is chosen as $S / A=30$ being characteristic to global (' $4 \pi$ ') SPS results [15]. Here we have chosen the ansatz (5)for the expansion of the volume being linear in time in the transverse direction. The parameter $v_{\text {lin }}$ is varied to simulate slow or fast expansion of the late hadronic fireball. The general characteristics is that first the antihyperons are dramatically being populated, and then in the very late expansion some more are still being annihilated, depending on how fast the expansion goes. A rapid expansion gives a higher yield, which can increase the final yield by a factor of 2 to 3 . However, the typical expansion behaviour obtained from simulations or extracted from the analysis of transverse momentum slopes of individual hadrons (pions and protons) is that at the late stages the transverse expansion velocity shoul be about $0.5 \mathrm{c}$. In the following we stay to the second ansatz (6) which extrapolates from an initially slower to a later faster expansion. 


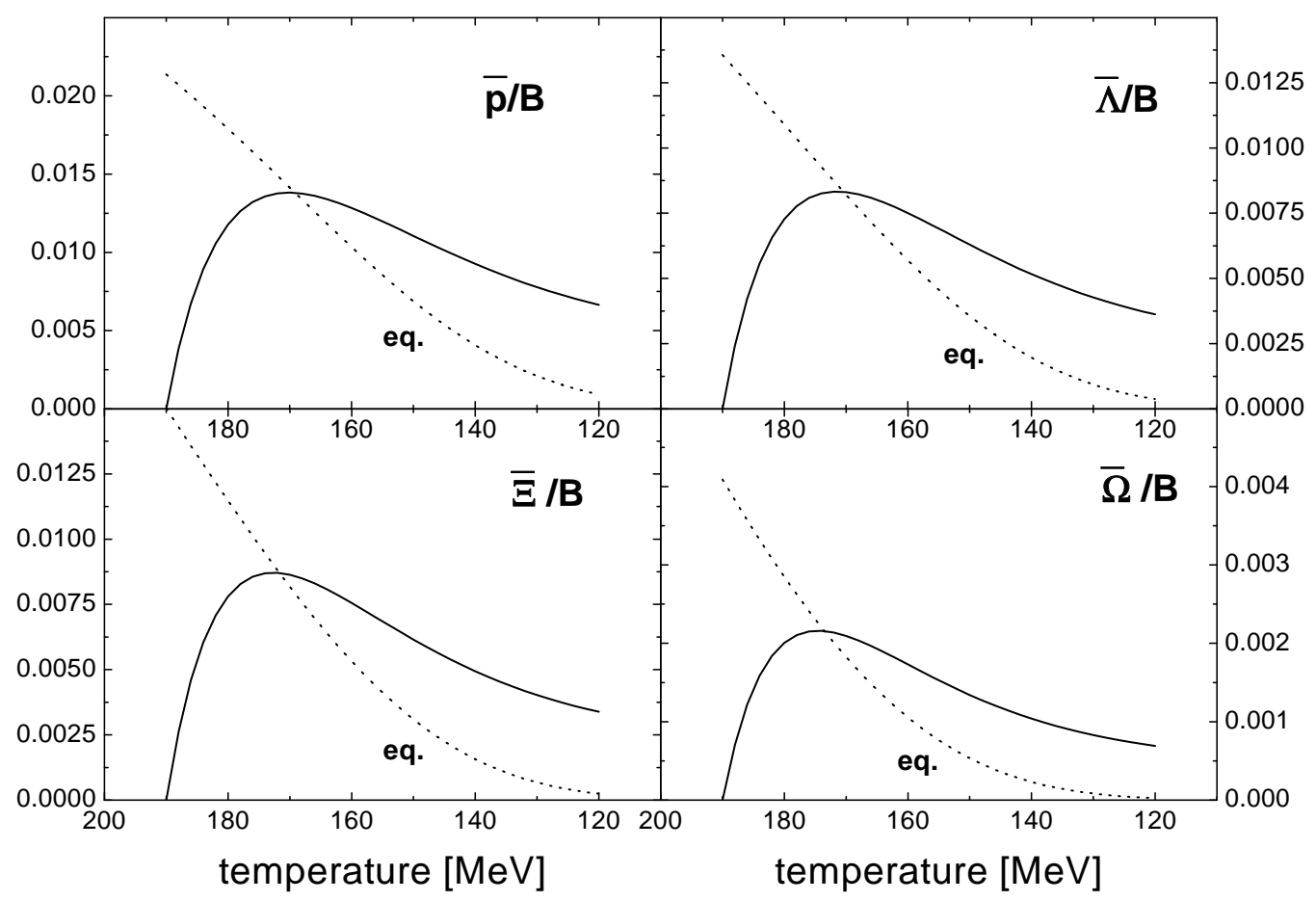

FIG. 6. The antihyperon to baryon number ratio $N_{\bar{Y}} / N_{B}(T)$ and $N_{\bar{Y}}^{e q .} / N_{B}(T)$ (dotted line) as a function of the decreasing temperature. Parameters are the same as in Fig. 5.

In Fig. 5 the number of $\bar{\Lambda} \mathrm{s}$ as a function of time is depicted, where now the cross section employed is varied by a constant factor, i.e. $\sigma_{e f f} \equiv \lambda \sigma_{0}$. The results are rather robust against a variation by a factor of 2 in the cross section. Typically (for $\lambda=1$ ) about more than 5 times in number of antihyperons are created during the evolution compared to the final number freezing out, thus reflecting the fast back ('annihilation') and forth ('creation') processes at work dictated by detailed balance. 


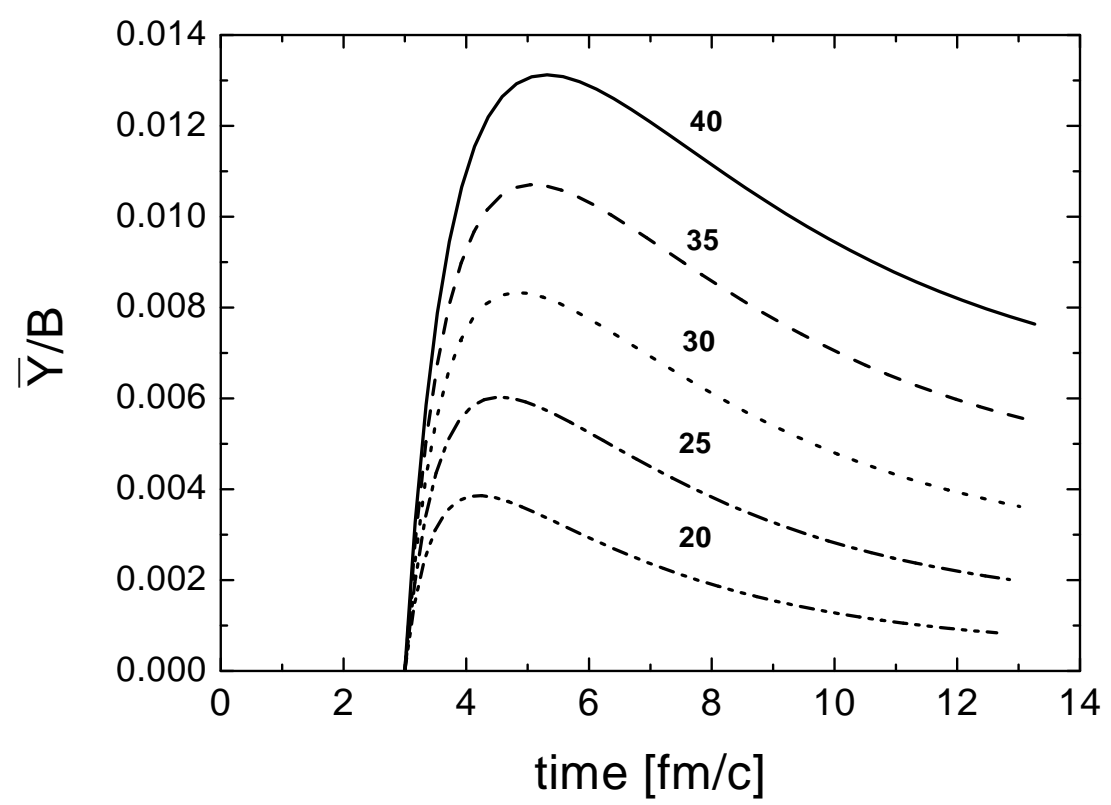

FIG. 7. $N_{\bar{\Lambda}} / N_{B}(t)$ as a function of time for various entropy content described via the entropy per baryon ratio $(S / A=20-40)$. Other parameters are as in Fig. 5.

In Fig. 6 the number of antihyperons of each specie are now shown as a function of the decreasing temperature $T(t)$ of the hadronic system. For a direct comparison the instantaneous equilibrium abundancy $N_{\bar{Y}}^{e q .}\left(T(t), \mu_{B}(t), \mu_{s}(t)\right) / N_{B}$ is also given. As noted above, after a fast initial population, the individual yields of the antihyperons do overshoot their respective equilibrium number and then do finally saturate at some slightly smaller value. Moreover, one notices that the yields effectively do saturate at a number which can be compared to an equivalent equilibrium number at a temperature parameter around $T_{\text {eff }} \approx 150-160$ $\mathrm{MeV}$, being strikingly close to the ones obtained within the various thermal analyses [3].

In Fig. 7 the number of anti- $\Lambda \mathrm{s}$ as a function of time is given for various entropy per baryon ratios. One notices that the final value in the yield significantly depends on the entropy content, or, in other words, on the baryochemical potential. We note that the results at midrapidity from WA97 can best be reproduced by employing an entropy to baryon ratio $S / A=40$. Indeed, at midrapidity one qualitatively expects a higher entropy content due to the larger pion to baryon ratio as compared to full ' $4 \pi$ ' data over all rapidities. At this point it will also be very interesting to compare our semi-quantitative calculations with the new results from NA49 on the $\bar{\Lambda}$-yield at lower SPS energies of $80 \mathrm{AGeV}$ and $40 \mathrm{AGeV}$ with lower entropy contents, respectively. As the presented results are very sensitive on the entropy content, one first needs a clean analysis to obtain a rather accurate $S / A$ number from the measured pion and proton abundancies. 


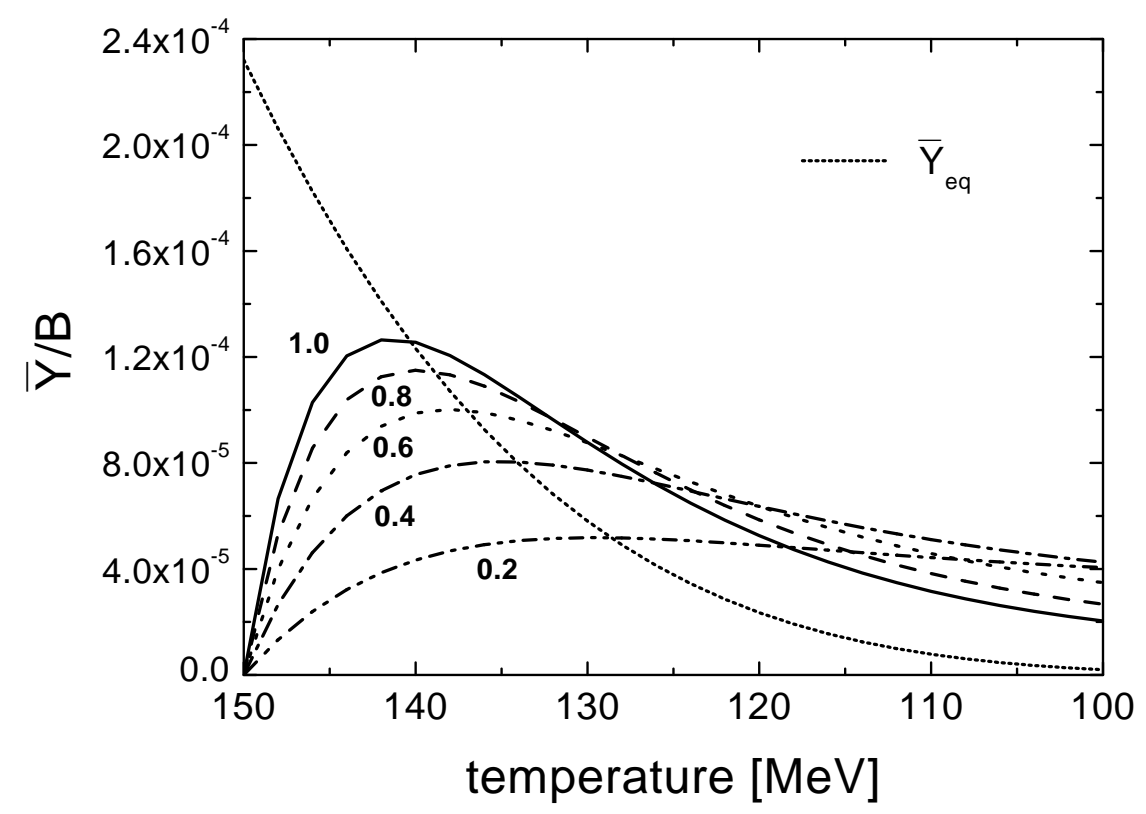

FIG. 8. $N_{\bar{\Lambda}} / N_{B}(T)$ and $N_{\bar{\Lambda}}^{e q .} / N_{B}(T)$ as a function of decreasing temperature for a characteristic AGS situation with an entropy content of $S / A=12$ for various implemented annihiation cross section $\sigma_{e f f} \equiv \lambda \sigma_{0} . t_{0}=5 \mathrm{fm} / \mathrm{c}$ and $T_{0}=150 \mathrm{MeV}$.

There is also a clear hint at AGS energies of enhanced anti- $\Lambda$ production [16]. For most central collisions more anti- $\Lambda$ s are found compared to anti-protons, which is quite puzzling as within a thermal model analysis this ratio is found not to be larger than 1 . This enhanced ratio of anti- $\Lambda$ s compared to anti-protons at AGS energies one can understand in a way that one assumes that their annihilation cross section on baryons is just slightly smaller than for the antiprotons. In Fig. 8 a similar study like that of Fig. 5 is shown for a characteristic situation at AGS. For smaller, yet not too small effective cross sections the final yield can here be enlarged by a factor of 2 compared to the case with a 'full' crossection, as the final reabsorption is not as effective. But, of course, this idea is speculation at present. Also, we remark that the $\bar{\Lambda}$ s effectively do saturate at an equivalent equilibrium number at a temperature parameter around $T_{\text {eff }} \approx 120-130 \mathrm{MeV}$. Unfortunately, there are no data for $\bar{\Xi}$ at AGS. Again the new NA49 data at lower energies are worthwhile to pursue. A detailed measurement of all antihyperons represents also an excellent opportunity for future heavy ion facilities at an energy upgraded GSI.

To summarize, multi-mesonic production of antihyperons is a consequence of detailed balance and, as the annihilation rate is large, it is by far the most dominant source in a hadronic gas. This is a remarkable observation, as it clearly demonstrates the importance of hadronic multi-particle channels, occuring frequently enough in a (moderately) dense hadronic environment in order to populate and chemically saturate the rare antibaryons. In order to be more competitive for a direct comparison with various experimental findings, new strategies have to be developed to describe for such multi-particle interactions within present day transport codes. A significant first step forward was very recently made; first results concerning the production of anti-protons at AGS and SPS energies are quite impressive 
[17]. Another strategy could be to exploit microscopically the concept of two meson doorway states (2) and their sequential decay by standard binary scattering processes. 


\section{REFERENCES}

[1] R. Stock, arXiv:hep-ph/0204032 .

[2] P. Koch, B. Müller and J. Rafelski, Phys. Rep. 142, 167 (1986); J. Rafelski, Phys. Lett. B 262, 333 (1991).

[3] P. Braun-Munzinger, I. Heppe and J. Stachel, Phys. Lett. B 465, 1 (1999); F. Becattini, J. Cleymans, A. Keränen, E. Suhonen and K. Redlich, PRC 64, 024901 (2001).

[4] H. Sorge et al, Phys. Lett. B 289, 6 (1992); H. Sorge, Z. Phys. C 67, 479 (1995); Phys. Rev. C 52, 3291 (1995); K. Werner and J. Aichelin, Phys. Lett. B 300, 158 (1993) and Phys. Lett. B 308, 372 (1993); N. Armesto, M.A. Braun, E.G. Ferreiro and C. Pajares, Phys. Lett. B 344, 301 (1995); E.G. Ferreiro and C. Pajares, Z. Phys. C 73, 309 (1997); M. Bleicher et al, Phys. Lett. B 485, 133 (2000).

[5] H. Sorge, Nucl. Phys. A 630, 522c (1998).

[6] C. Greiner and S. Leupold, J. Phys. G 27, L95 (2001); C. Greiner, arXiv:nuclth/0011026 ; Nucl. Phys. A 698, 591 (2002).

[7] R. Rapp and E. Shuryak, Phys. Rev. Lett. 86, 2980 (2001).

[8] J. Vandermeulen, PRC 33, 1101 (1986).

[9] F. Eisele et al, Phys. Lett. B 60, 297 (1976); G.J. Wang, G. Welke, R. Bellwied and C. Pruneau, arXiv:nucl-th/9807036.

[10] J. Vandermeulen, ZPC 37, 563 (1988); J. Cugnon and J. Vandermeulen, PRC 39, 181 (1989).

[11] C. Dover, T. Gutsche, M. Maruyama and A. Faessler, Prog. Part. Nucl. Phys. 29, 87 (1992).

[12] J. Geiss, W. Cassing and C. Greiner, Nucl. Phys. A 644, 107 (1998).

[13] W. Cassing, private communication.

[14] C. Greiner, J. Phys. G 28, 1631 (2002).

[15] J. Cleymans, B. Kämpfer and S. Wheaton, arXiv:nucl-th/0110035 .

[16] L. Ahle et al, (E-802 Collaboration), Phys. Rev. Lett. 81, 2650 (1998); B. Back et al, (E-917 Collaboration), Phys. Rev. Lett. 87, 242301 (2001).

[17] W. Cassing, Nucl. Phys. A 700, 618 (2002). 


\title{
Importance of multi-mesonic fusion processes on (strange) antibaryon production
}

\author{
C. GREINER \\ Institut für Theoretische Physik, Universität Giessen, Heinrich-Buff-Ring 16, D-35392 Giessen, \\ Germany
}

\begin{abstract}
Sufficiently fast chemical equilibration of (strange) antibaryons in an environment of nucleons, pions and kaons during the course of a relativistic heavy ion collision can be understood by a 'clustering' of mesons to buildt up baryon-antibaryon pairs. This multi-mesonic (fusion-type) process has to exist in medium due to the principle of detailed balance. Novel numerical calculations for a dynamical setup are presented. They show that - at maximum SPS energies - yields of each antihyperon specie are obtained which are consistent with chemical saturated populations of $T \approx 150-160 \mathrm{MeV}$, in line with popular chemical freeze-out parameters extracted from thermal model analyses.
\end{abstract}

\section{BRIEF OVERVIEW ON ANTIHYPERON PRODUCTION}

Strangeness enhancement has been predicted a long time ago as a potential probe to find clear evidence for the temporary existence of a quark gluon plasma (QGP) in relativistic heavy ion collisions. A strong experimental effort has been made since and is still made for measuring strange particle abundancies in experiments at Brookhaven and at CERN (for a short recent review see [1]). In particular because of high production thresholds in binary hadronic reaction channels antihyperons had been advocated as the appropriate QGP candidates [2]. Indeed, a satisfactory picture of nearly chemically saturated populations of antihyperons has been experimentally demonstrated over the last years with the $\mathrm{Pb}+\mathrm{Pb}$ experiments NA49 and WA97 at CERN-SPS. For this statement, of course, a quantitative, theoretical analysis by employing a thermal (or 'statistical') model has to be invoked by fitting the thermodynamical parameters to the set of individual (strange) hadronic abundancies [3].

On the other hand, already since the first measurements with the lighter ions have been undertaken in the early nineties, the theoretical description of the antibaryon production within hadronic transport schemes in comparison to these data faced some severe difficulties. Some phenomenological motivated attempts to explain a more abundant production of antihyperons within a hadronic transport description [4] had been proposed like the appearance of color ropes, the fusion of strings, the percolation of strings, or the formation of high-dense hadronic clusters. The underlying mechanisms, however, have to be considered exotic and to some extent ad hoc, their purpose lies mainly to create (much) more antibaryon in the very early intial stage of the reaction (compared to simple rescaled $p+p$ collisions). To some extent indeed the philosophy behind these mechanisms was to model a precursor of initial QGP formation within a hadronic trans- 


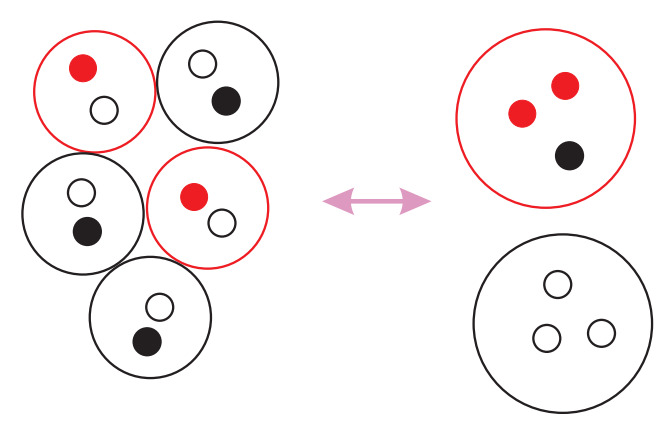

FIGURE 1. Schematic picture for the multi-mesonic fusion-like reaction $3 \pi+2 K \leftrightarrow \bar{\Xi}+N$.

port scheme. On the other hand, in most of the transport calculations a dramatic role of subsequent antibaryon annihilation is observed, which, in return, has to be more than counterbalanced by these more exotic, initially occuring mechanisms. Large annihilation rates result if the free-space cross section is employed. The situation seems even more paradox with respect to the fact that the chemical description within thermal models works indeed amazingly well for the antihyperons and being, of course, completely nondependent on the (large) magnitude of the annihilation cross section. For all of this reasons the theoretical and dynamical understanding of the production of (strange) antibaryons has remained a delicate and challenging task [5].

As we will demonstrate, a correct incorporation of the baryonic annihilation channels had actually not been done consistently. We have conjectured recently that a sufficiently fast redistributions of strange and light quarks into (strange) baryon-antibaryon pairs should be achieved by multi-mesonic fusion-type reactions of the type

$$
n_{1} \pi+n_{2} K \leftrightarrow \bar{Y}+p
$$

occuring in a moderately dense hadronic system [6] (illustrated in Fig. 1). The beauty of this argument lies in the fact that (at least) these special kind of multi-hadronic reactions have to be present because of the fundamental principle of detailed balance. As the annihilation of antihyperons on baryons is of dramatic relevance, the multi-mesonic (fusion-like) 'back-reactions' involving $n_{1}$ pions and $n_{2}$ kaons, where $n_{2}$ counts the number of anti-strange quarks within the antihyperon $\bar{Y}$ ), must, in principle, be taken care of in a dynamical simulation. These reactions are then the most dominant source of production. This crucial fact had been overseen in all of the aforementioned treatments. The underlying reasoning was first raised by Rapp and Shuryak who described the maintenance of nearly perfect chemical equilibrium of antiprotons together with pions and nucleons during the very late stage of the expanding fireball before the particles loose contact [7].

The crucial input, though plausible, is now to assume that the annihilation cross sections for any strange or nonstrange antibaryon on any baryon are approximately the same order as for $N \bar{p}$ at the same relative momenta, i.e. $\sigma_{B \bar{Y} \rightarrow n \pi+n_{Y} K} \approx \sigma_{N \bar{p} \rightarrow n \pi}$, being in the range of 50-100 mb for characteristic and moderately low momenta occuring in an expanding hadronic fireball. The equilibration timescale $\left(\Gamma_{\bar{Y}}\right)^{(-1)} \sim 1 /\left(\sigma_{B \bar{Y}} v_{B \bar{Y}} \rho_{B}\right)$ is to a good approximation proportional to the inverse of the density of baryons and 
their resonances. Adopting an initial density of 1-2 times normal nuclear matter density $\rho_{0}$ for the initial and thermalized hadronic fireball, the antihyperons do equilibrate on a timescale of 1-3 fm/c well within the expansion timescale of the late hadronic fireball. Hence, fast chemical equilibration of the antihyperon abundancies is guaranteed by detailed balance with respect to the strong annihilation, the final yields then being independent of the actual size of the (large) annihilation cross section, solving the aforementioned paradox.

To be quantitative, (some) novel results by solving rate calculations for a dynamical setup are presented in the following. Before turning to their discussion, we will review briefly on some general ideas of the baryon-antibaryon annihilation process, in order to strengthen the one main assumption concerning the general size of its cross section, and on how to come to general master equations.

\section{ANTIBARYON ANNIHILATION AND ITS EFFECTIVE DESCRIPTION BY A MASTER EQUATION}

Antinucleon-nucleon annihilation is the strongest of all strong interaction processes. The strong annihilation of a nucleon and an antinucleon can be thought quantum mechanically as a complete absorbtive scattering process given approximately by the black disk formula $\sigma_{a b s}=\pi(R+\lambda)^{2}$ or by a more sophisticated boundary condition description [8]. For $p+\bar{p}$-annihilation one finds for the 'black disk' radius $R=1.07 \mathrm{fm}$, for which then this decription reproduces very accurately the total inelastic cross section as a function of the beam momentum [8] and also its steep increase and diverging behaviour at low momenta. Hence, the quantum mechanical interpretation of the annihilation (being exotherm) is a picture of complete absorption. One is now tempted to generalize this simple and intuitive picture for all baryon-antibaryon annihilation processes. The only 'free' parameter which then can change is the radius $R$. Again, as it basically reflects the radius of the proton in the case of $p \bar{p}$-annihilation, one would expect that this canonical value of $1 \mathrm{fm}$ is of general validity for all the annihilation processes. Thus it is plausible to assume that the cross section for annihilation between any baryon and antibaryon should be rather the same for the same relative momenta. Indeed, there does exist old data on the total $\bar{\Lambda}+p$ cross section [9] with reasonably large value, although the $\bar{\Lambda}$ momenta was exceeding $4 \mathrm{GeV} / \mathrm{c}$ in all data taken. (These data are not conclusive to really extrapolate dwn to lower momenta, though they give a right indication: The authors of [9] reported an extrapolated formula of $\sigma_{a b s}=47( \pm 10) p_{\Lambda}^{-1 / 2} \mathrm{mb} \mathrm{GeV}^{-1 / 2}$, which is not fully analogue to the black disk formula, but has already a rather correct shape with a sizeable magnitude.)

A more microscopic and quite popular picture, which can decribe quantitatively the complicated final states of individual outgoing mesonic channels, is the concept of two meson doorway states [10] given by

$$
\bar{B}+B \rightarrow M_{1}+M_{2} \rightarrow n_{1} \pi+n_{2} K .
$$

The main assumption is that the annihilation occurs exclusively via two mesons with nearest threshold dominance. $M_{1}$ and $M_{2}$ can be rather highlying resonances and/or 
hybrid states which come close with their individual threshold. The final decay of these two mesons into the various channels of multiple pions and kaons is then treated statistically and microcanonically. It turns out that such a description describes rather nicely reproduces the available data and can also be generalized straightforwardly for antihyperon annihilation [10]. There do exist a lot of other phenomenological and microscopic descriptions like quark models or flux tube models, which try to describe quantitatively the complicated process of the annihilation in more dynamical terms (for a review see [11]).

How can one incorporate such complicated processes into a description of transport dynamics? It is clear that as the very microscopy of the annihilation processes is not fully understood, one has to abandon any detailed local modelling, but has to turn to an effective coarse grained description which is guided by physical principles. Following the concepts of relativistic kinetic theory, the microscopic starting point is a Boltzmanntype equation of the form

$$
\begin{aligned}
\partial_{t} f_{\bar{Y}}+\frac{\mathbf{p}}{E_{\bar{Y}}} \nabla f_{\bar{Y}}= & \sum_{\left\{n_{1}\right\} ; B} \frac{1}{2 E_{\bar{Y}}} \int \frac{d^{3} p_{B}}{(2 \pi)^{3} 2 E_{B}} \prod_{\left\{n_{1}, n_{2}\right\}} \int \frac{d^{3} p_{i}}{(2 \pi)^{3} 2 E_{i}}(2 \pi)^{4} \delta^{4}\left(p_{\bar{Y}}+p_{B}-\sum_{\left\{n_{1}, n_{2}\right\}} p_{i}\right) \\
& \left|\left\langle\left\langle n_{1}, n_{2}|T| \bar{Y} B\right\rangle\right\rangle\right|^{2}\left\{(-) f_{\bar{Y}} f_{B} \prod_{\left\{n_{1}, n_{2}\right\}}\left(1+f_{i}\right)+\prod_{\left\{n_{1}, n_{2}\right\}} f_{i}\left(1-f_{\bar{Y}}\right)\left(1-f_{B}\right)\right\},
\end{aligned}
$$

where

$$
\sigma_{\bar{Y} B}^{\left\{n_{1}\right\}} \equiv \frac{1}{{ }^{6} F l u x^{\prime}} \sum_{\left\{n_{1}\right\}} \prod_{\left\{n_{1}, n_{2}\right\}} \int \frac{d^{3} p_{i}}{(2 \pi)^{3} 2 E_{i}}(2 \pi)^{4} \delta^{4}\left(p_{\bar{Y}}+p_{B}-\sum_{\left\{n_{1}, n_{2}\right\}} p_{i}\right)\left|\left\langle\left\langle n_{1}, n_{2}|T| \bar{Y} B\right\rangle\right\rangle\right|^{2}
$$

corresponds to the total annihilation cross section into the various possible multiple pion states. This form of the collision exactly incorporates the principle of detailed balance. The static fixed point of this equation (together with the usual binary kinetic processes) are thermal and chemical saturated distributions. Without the back reaction channels the equations would only have vanishing distributons as stable fixed points, which is, of course, unacceptable. Hence, the back reactions have to be considered as very basic and important ingredient of the transport description when trying to implement the baryonantibaryon annihilation processes.

Furthermore it is obvious that the back reactions will guarantee that the (strange) antibaryons become chemical saturated with the pions, kaons and nucleons on a very short timescale. To see this more explicit, and also for the further numerical treatment, one can bring the above Boltzmann equation into a more intuitive form of a master or rate equation. Assuming $v_{r e l} \sigma_{\bar{Y} B}(\sqrt{s})$ to be roughly constant, which is actually a good approximation for the $p \bar{p}$-annihilation, or, invoking a standard description of further effective coarse graining by using thermally averaged cross sections and distributions, and furthermore taking the distributions in the Boltzmann approximantion, the following master equation for the respectively considered antihyperon density is obtained

$$
\frac{d}{d t} \rho_{\bar{Y}}=-\left\langle\left\langle\sigma_{\bar{Y} B} v_{\bar{Y} B}\right\rangle\right\rangle\left\{\rho_{\bar{Y}} \rho_{B}-\sum_{\left\{n_{1}\right\}} \hat{M}_{\left(n_{1}, n_{2}\right)}\left(T, \mu_{B}, \mu_{s}\right)\left(\rho_{\pi}\right)^{n_{1}}\left(\rho_{K}\right)^{n_{2}}\right\},
$$




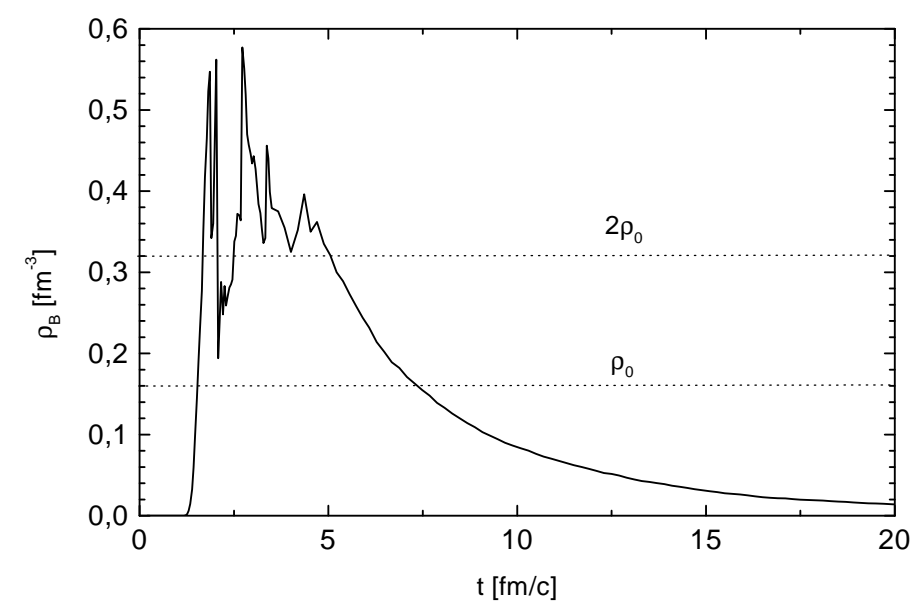

FIGURE 2. Time evolution of the (average) net baryon density for midrapidity $|\Delta Y| \leq 1$ and central $\mathrm{Pb}+\mathrm{Pb}$-collision at $160 \mathrm{AGeV}$ obtained within a dynamical transport simulation. Here the amount of baryon number residing still in string-like excitations is explicitely discarded. String-like excitations have disappeared after about 3-4 fm/c, so that from this time on a pure hadronic fireball develops and expands. Its initial net baryon-density starts slightly above $\rho_{B}=2 \rho_{0}$.

where the 'back-reactions' of several effectively clustering pions and kaons are incorporated in the 'mass-law' factor

$$
\hat{M}_{\left(n_{1}, n_{2}\right)}\left(T, \mu_{B}, \mu_{s}\right)=\frac{\rho_{\bar{Y}}^{e q .} \rho_{B}^{e q .}}{\left(\rho_{\pi}^{e q \cdot}\right)^{n_{1}}\left(\rho_{K}^{e q \cdot}\right)^{n_{2}}} p_{n_{1}} .
$$

Here $p_{n_{1}}$ (which will generally depend on the thermodynamical parameters) states the relative probability of the reaction (1) to decay into a specific number $n_{1}$ of pions and $\rho_{B}$ denotes the total number density of baryonic particles. As is well-known, the mass-law factor $\hat{M}$ depends only on the temperature and the baryon and strange quark chemical potentials. $\Gamma_{\bar{Y}} \equiv\left\langle\left\langle\sigma_{\bar{Y} N} v_{\bar{Y} N}\right\rangle\right\rangle \rho_{B}$ gives the effective annihilation rate of the respective antihyperon specie on a baryon.

For a further manipulation one has to make assumptions for the various abundancies occuring in the master equation. Nonequilibrium inelastic hadronic reactions can explain to a good extent the overall strangeness production seen experimentally: The major amount of the produced kaons at SPS-energies can be understood in terms of still early and energetic non-equilibrium interactions [12]. In Fig. 2 the time evolution of netbaryon density at midrapidiy obtained within a dynamical transport simulation [13] is depicted. All strangeness is being produced still when string-like excitations are governing the dynamics. In the later hadronic stage the number of strange quarks stays more or less constant and can only be redistributed among the various hadrons [14]. Also the pions and nucleons do stay more or less at thermal equilibrium. Refering to the master equation (3), one can then take the pions, baryons and kaons to stay approximately in thermal equilibrium throughout the later hadronic evolution of the collision, the later being modelled to be an isentropic expansion with fixed total entropy content being specified 


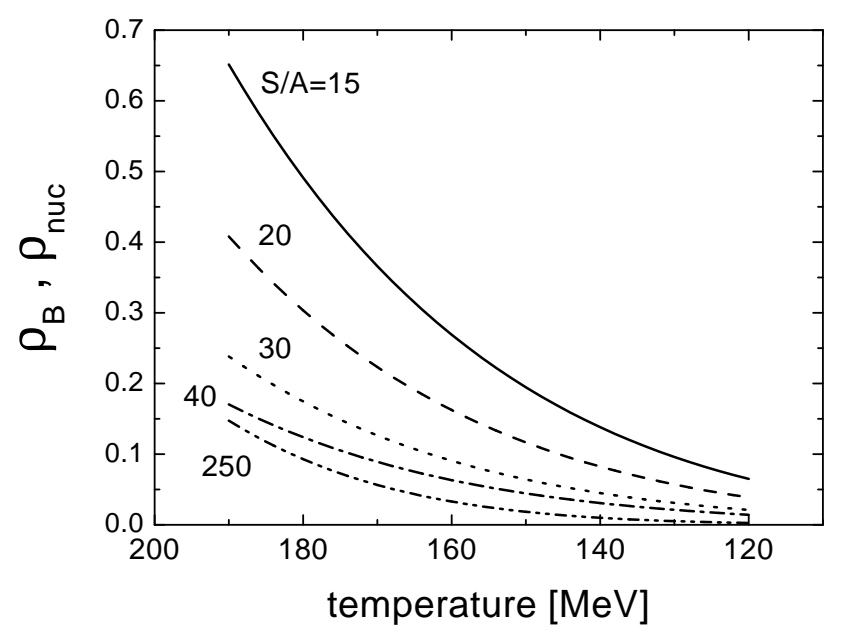

FIGURE 3. The netbaryon density $\rho_{B}$ of a chemically fully equilibrated thermal hadronic resonance gas as function of decreasing temperature for the situation of an isentropic expansion, i.e. for various constant entropy/baryon ratios. For the value $S / A=250$ (a situation expected at RHIC) the total baryon number density $\rho_{\text {nuc }}$ is plotted instead.

via the entropy per baryon ratio $S / A$ (compare with Fig. 3). (3) has the intuitive form

$$
\frac{d}{d t} \rho_{\bar{Y}}=-\Gamma_{\bar{Y}}\left\{\rho_{\bar{Y}}-\rho_{\bar{Y}}^{e q}\right\}
$$

The production rate per unit volume $d N_{\bar{Y}} / d t d V$ is given by the non-vanishing, though small value $\Gamma_{\bar{Y}} \rho_{\bar{Y}}^{e q}$. Still this rate is enough to populate the antihyperons to their (very small) equilibrium value.

For the calculations solving this master equation (4) one has to employ an 'effective' volume $V(t)$ in order to extrapolate from Fig. 2 to Fig. 3, i.e. simulating the global characteristic of the expansion and the dilution of the baryon density and thus the annihilation rate $\Gamma_{\bar{Y}}(t)$. The 'effective' (global or at midrapidity) volume $V(t)$ is parametrized as function of time by longitudinal Bjorken expansion and including a transversal expansion either with a linear profile

$$
V_{e f f, \text { lin }}\left(t \geq t_{0}\right)=\pi(c t)\left(R_{0}+v_{\text {lin }}\left(t-t_{0}\right)\right)^{2}
$$

(taking $v_{\text {lin }}$ as an appropriate parameter to simulate slower or faster expansion) or with an accelerating radial flow

$$
V_{e f f, a c c}\left(t \geq t_{0}\right)=\pi(c t)\left(R_{0}+v_{0}\left(t-t_{0}\right)+0.5 a_{0}\left(t-t_{0}\right)^{2}\right)^{2}
$$

with $R_{0}=6.5 \mathrm{fm}, v_{0}=0.15 \mathrm{c}$ and $a_{0}=0.05 \mathrm{c}^{2} / \mathrm{fm}$ for the later modeling. 


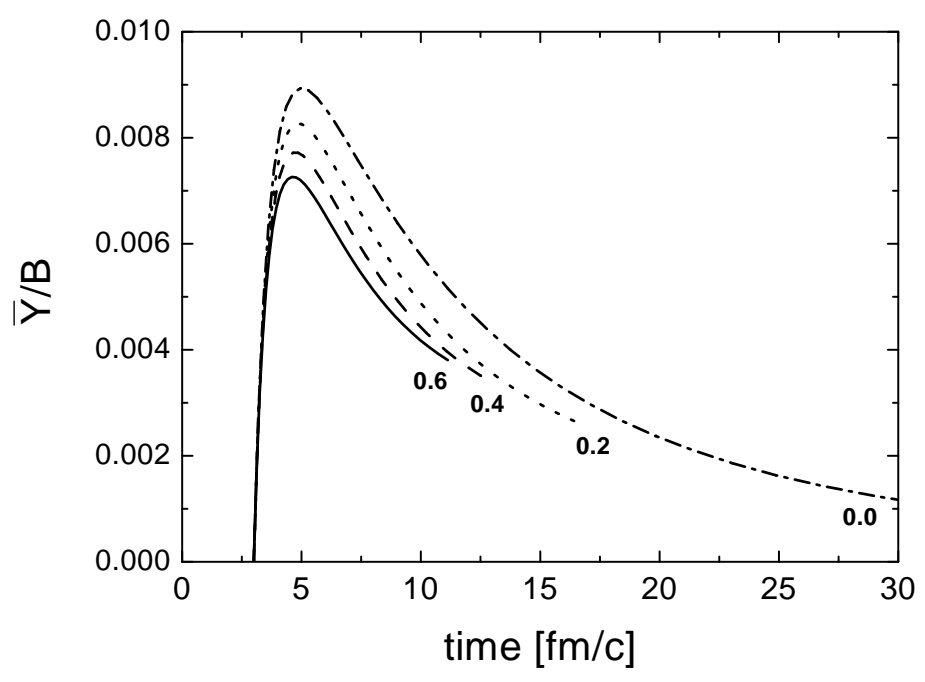

FIGURE 4. The anti- $\Lambda$ to baryon number ratio $N_{\bar{\Lambda}} / N_{B}(t)$ as a function of time for various velocity parameters $v_{\text {lin }}$ for the transverse expansion. The entropy per baryon is taken as $S / A=30, t_{0}=3 \mathrm{fm} / \mathrm{c}$ and $T_{0}=190 \mathrm{MeV}$.

\section{RESULTS AND IMPLICATIONS}

At starting time $t_{0}$ an initial temperature $T_{0}$ is chosen. ( $T_{0}$ is set to $190 \mathrm{MeV}$ for the SPS and $150 \mathrm{MeV}$ for the AGS situation, while the initial energy densities are then about $1 \mathrm{GeV} / \mathrm{fm}^{3}$.) From (5) or (6) together with the constraint of conserved entropy the temperature and the chemical potentials do follow as function of time and thus also $\rho_{B}(t)$ as well as $\rho_{\bar{Y}}^{e q}(t)$ within the hadronic resonance gas. As a minimal assumption the initial abundancy of antihyperons is set to zero. Equation (4), taking into account the volume dilution, is solved for each specie. For the thermally averaged cross section we take a simple constant value of $\left\langle\sigma_{a n n} v\right\rangle:=\sigma_{0} \equiv 40 \mathrm{mb}$.

In Fig. 4 the number of $\bar{\Lambda}$ s (normalized to the conserved net baryon number) as a function of time is depicted. The entropy per baryon is chosen as $S / A=30$ being characteristic to global (' $4 \pi$ ') SPS results [15]. Here we have chosen the ansatz (5)for the expansion of the volume being linear in time in the transverse direction. The parameter $v_{\text {lin }}$ is varied to simulate slow or fast expansion of the late hadronic fireball. The general characteristics is that first the antihyperons are dramatically being populated, and then in the very late expansion some more are still being annihilated, depending on how fast the expansion goes. A rapid expansion gives a higher yield, which can increase the final yield by a factor of 2 to 3 . However, the typical expansion behaviour obtained from simulations or extracted from the analysis of transverse momentum slopes of individual hadrons (pions and protons) is that at the late stages the transverse expansion velocity shoul be about $0.5 \mathrm{c}$. In the following we stay to the second ansatz (6) which extrapolates from an initially slower to a later faster expansion.

In Fig. 5 the number of $\bar{\Lambda}$ s as a function of time is depicted, where now the cross 


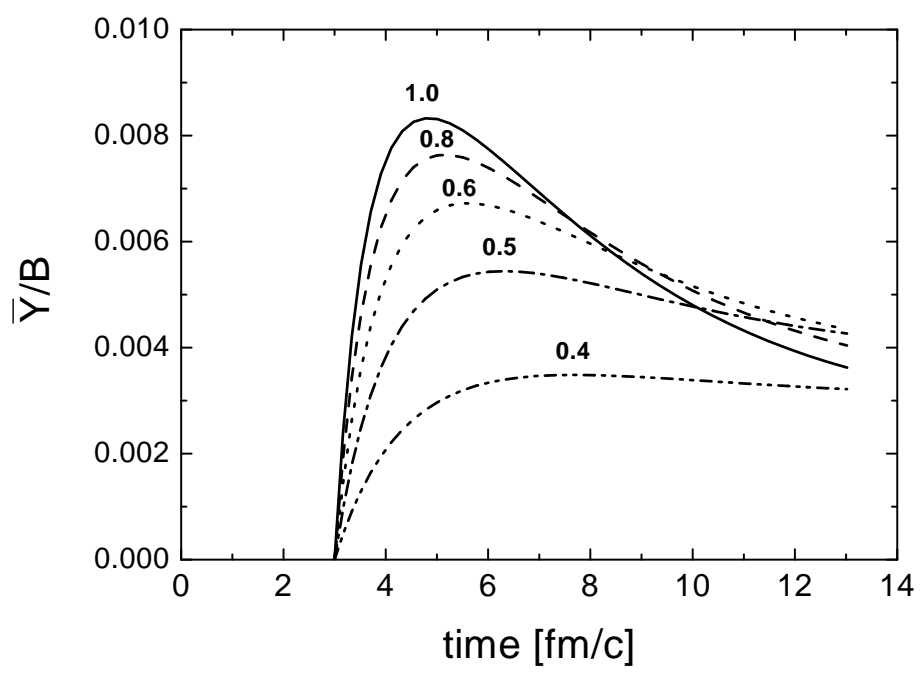

FIGURE 5. The anti- $\Lambda$ to baryon number ratio $N_{\bar{\Lambda}} / N_{B}(t)$ as a function of time for various implemented annihiation cross section $\sigma_{\text {eff }} \equiv \lambda \sigma_{0}$. The entropy per baryon is taken as $S / A=30, t_{0}=3 \mathrm{fm} / \mathrm{c}$ and $T_{0}=190 \mathrm{MeV}$.

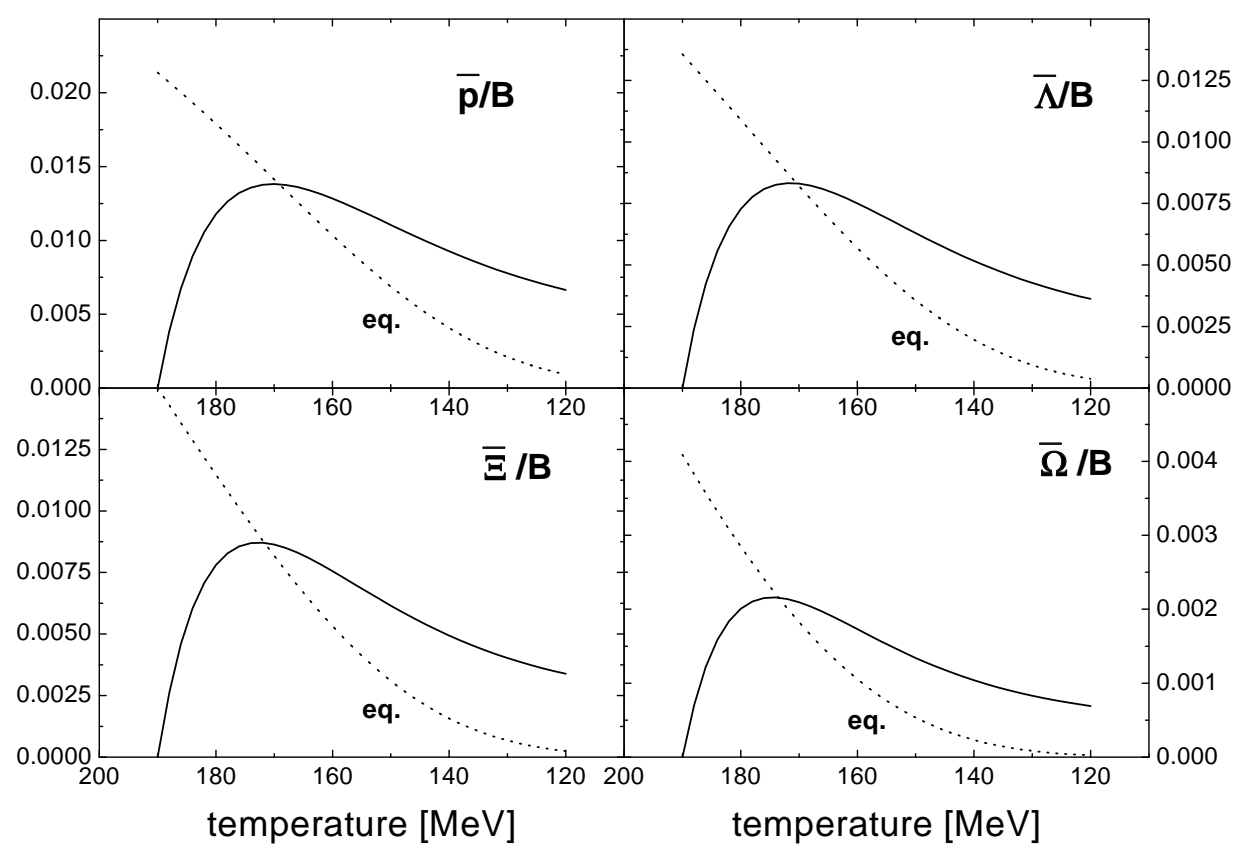

FIGURE 6. The antihyperon to baryon number ratio $N_{\bar{Y}} / N_{B}(T)$ and $N_{\bar{Y}}^{e q .} / N_{B}(T)$ (dotted line) as a function of the decreasing temperature. Parameters are the same as in Fig. 5. 


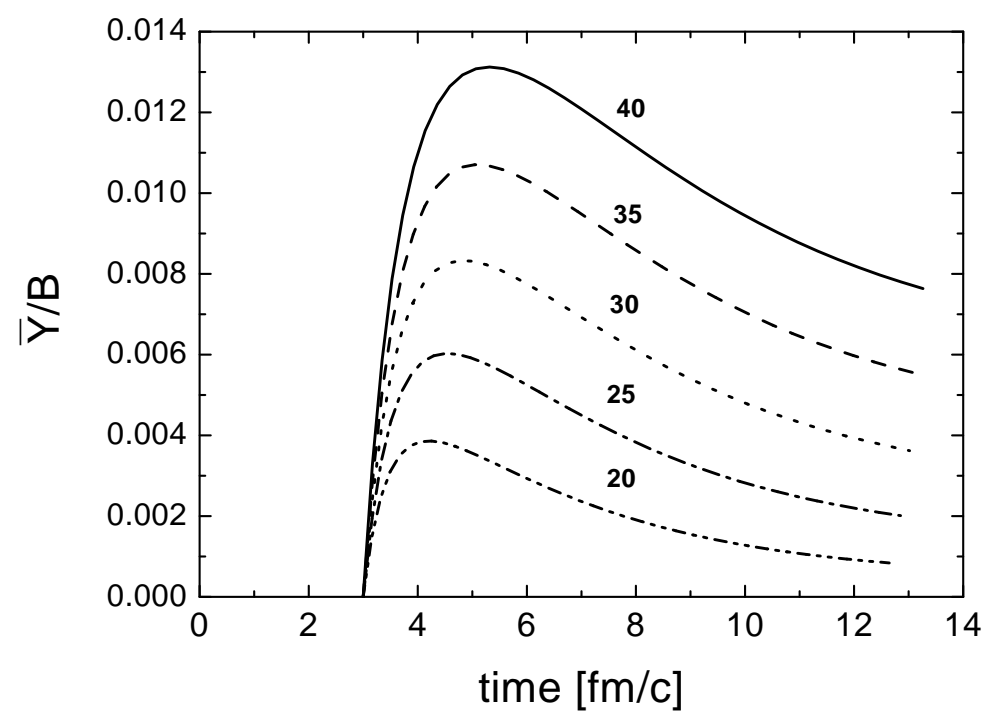

FIGURE 7. $N_{\bar{\Lambda}} / N_{B}(t)$ as a function of time for various entropy content described via the entropy per baryon ratio $(S / A=20-40)$. Other parameters are as in Fig. 5.

section employed is varied by a constant factor, i.e. $\sigma_{e f f} \equiv \lambda \sigma_{0}$. The results are rather robust against a variation by a factor of 2 in the cross section. Typically (for $\lambda=1$ ) about more than 5 times in number of antihyperons are created during the evolution compared to the final number freezing out, thus reflecting the fast back ('annihilation') and forth ('creation') processes at work dictated by detailed balance.

In Fig. 6 the number of antihyperons of each specie are now shown as a function of the decreasing temperature $T(t)$ of the hadronic system. For a direct comparison the instantaneous equilibrium abundancy $N_{\bar{Y}}^{e q} \cdot\left(T(t), \mu_{B}(t), \mu_{s}(t)\right) / N_{B}$ is also given. As noted above, after a fast initial population, the individual yields of the antihyperons do overshoot their respective equilibrium number and then do finally saturate at some slightly smaller value. Moreover, one notices that the yields effectively do saturate at a number which can be compared to an equivalent equilibrium number at a temperature parameter around $T_{\text {eff }} \approx 150-160 \mathrm{MeV}$, being strikingly close to the ones obtained within the various thermal analyses [3].

In Fig. 7 the number of anti- $\Lambda$ s as a function of time is given for various entropy per baryon ratios. One notices that the final value in the yield significantly depends on the entropy content, or, in other words, on the baryochemical potential. We note that the results at midrapidity from WA97 can best be reproduced by employing an entropy to baryon ratio $S / A=40$. Indeed, at midrapidity one qualitatively expects a higher entropy content due to the larger pion to baryon ratio as compared to full ' $4 \pi$ ' data over all rapidities. At this point it will also be very interesting to compare our semi-quantitative calculations with the new results from NA49 on the $\bar{\Lambda}$-yield at lower SPS energies of 80 $\mathrm{AGeV}$ and $40 \mathrm{AGeV}$ with lower entropy contents, respectively. As the presented results are very sensitive on the entropy content, one first needs a clean analysis to obtain a 


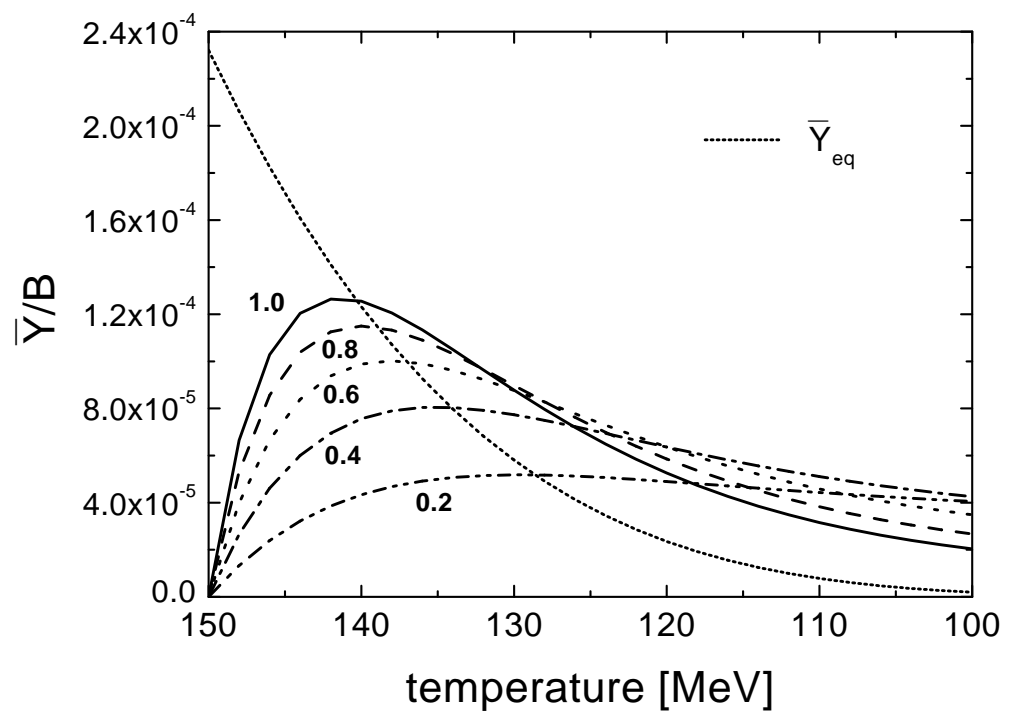

FIGURE 8. $\quad N_{\bar{\Lambda}} / N_{B}(T)$ and $N_{\bar{\Lambda}}^{e q .} / N_{B}(T)$ as a function of decreasing temperature for a characteristic AGS situation with an entropy content of $S / A=12$ for various implemented annihiation cross section $\sigma_{\text {eff }} \equiv \lambda \sigma_{0} \cdot t_{0}=5 \mathrm{fm} / \mathrm{c}$ and $T_{0}=150 \mathrm{MeV}$.

rather accurate $S / A$ number from the measured pion and proton abundancies.

There is also a clear hint at AGS energies of enhanced anti- $\Lambda$ production [16]. For most central collisions more anti- $\Lambda$ s are found compared to anti-protons, which is quite puzzling as within a thermal model analysis this ratio is found not to be larger than 1. This enhanced ratio of anti- $\Lambda$ s compared to anti-protons at AGS energies one can understand in a way that one assumes that their annihilation cross section on baryons is just slightly smaller than for the antiprotons. In Fig. 8 a similar study like that of Fig. 5 is shown for a characteristic situation at AGS. For smaller, yet not too small effective cross sections the final yield can here be enlarged by a factor of 2 compared to the case with a 'full' crossection, as the final reabsorption is not as effective. But, of course, this idea is speculation at present. Also, we remark that the $\bar{\Lambda}$ s effectively do saturate at an equivalent equilibrium number at a temperature parameter around $T_{\text {eff }} \approx 120-130 \mathrm{MeV}$. Unfortunately, there are no data for $\bar{\Xi}$ at AGS. Again the new NA49 data at lower energies are worthwhile to pursue. A detailed measurement of all antihyperons represents also an excellent opportunity for future heavy ion facilities at an energy upgraded GSI.

To summarize, multi-mesonic production of antihyperons is a consequence of detailed balance and, as the annihilation rate is large, it is by far the most dominant source in a hadronic gas. This is a remarkable observation, as it clearly demonstrates the importance of hadronic multi-particle channels, occuring frequently enough in a (moderately) dense hadronic environment in order to populate and chemically saturate the rare antibaryons. In order to be more competitive for a direct comparison with various experimental findings, new strategies have to be developed to describe for such multi-particle interactions 
within present day transport codes. A significant first step forward was very recently made; first results concerning the production of anti-protons at AGS and SPS energies are quite impressive [17]. Another strategy could be to exploit microscopically the concept of two meson doorway states (2) and their sequential decay by standard binary scattering processes.

\section{REFERENCES}

1. R. Stock, arXiv:hep-ph/0204032 .

2. P. Koch, B. Müller and J. Rafelski, Phys. Rep. 142, 167 (1986); J. Rafelski, Phys. Lett. B 262, 333 (1991).

3. P. Braun-Munzinger, I. Heppe and J. Stachel, Phys. Lett. B 465, 1 (1999); F. Becattini, J. Cleymans, A. Keränen, E. Suhonen and K. Redlich, PRC 64, 024901 (2001).

4. H. Sorge et al, Phys. Lett. B 289, 6 (1992); H. Sorge, Z. Phys. C 67, 479 (1995); Phys. Rev. C 52, 3291 (1995); K. Werner and J. Aichelin, Phys. Lett. B 300, 158 (1993) and Phys. Lett. B 308, 372 (1993); N. Armesto, M.A. Braun, E.G. Ferreiro and C. Pajares, Phys. Lett. B 344, 301 (1995); E.G. Ferreiro and C. Pajares, Z. Phys. C 73, 309 (1997); M. Bleicher et al, Phys. Lett. B 485, 133 (2000).

5. H. Sorge, Nucl. Phys. A 630, 522c (1998).

6. C. Greiner and S. Leupold, J. Phys. G 27, L95 (2001); C. Greiner, arXiv:nucl-th/0011026 ; Nucl. Phys. A 698, 591 (2002).

7. R. Rapp and E. Shuryak, Phys. Rev. Lett. 86, 2980 (2001).

8. J. Vandermeulen, PRC 33, 1101 (1986).

9. F. Eisele et al, Phys. Lett. B 60, 297 (1976); G.J. Wang, G. Welke, R. Bellwied and C. Pruneau, arXiv:nucl-th/9807036.

10. J. Vandermeulen, ZPC 37, 563 (1988); J. Cugnon and J. Vandermeulen, PRC 39, 181 (1989).

11. C. Dover, T. Gutsche, M. Maruyama and A. Faessler, Prog. Part. Nucl. Phys. 29, 87 (1992).

12. J. Geiss, W. Cassing and C. Greiner, Nucl. Phys. A 644, 107 (1998).

13. W. Cassing, private communication.

14. C. Greiner, J. Phys. G 28, 1631 (2002).

15. J. Cleymans, B. Kämpfer and S. Wheaton, arXiv:nucl-th/0110035 .

16. L. Ahle et al, (E-802 Collaboration), Phys. Rev. Lett. 81, 2650 (1998); B. Back et al, (E-917 Collaboration), Phys. Rev. Lett. 87, 242301 (2001).

17. W. Cassing, Nucl. Phys. A 700, 618 (2002). 